\title{
Optimal PMU Placement in Multi-configuration Power Distribution Networks
}

Van-khoi TRAN ${ }^{1,}$ a , He-sheng ZHANG ${ }^{2, \text { b }}$, Van-nghia NGUYEN ${ }^{3, c}$

${ }^{1}$ School of Electrical Engineering, Beijing Jiaotong University, Beijing 100044, China

${ }^{2}$ School of Electrical Engineering, Beijing Jiaotong University, Beijing 100044, China

${ }^{3}$ Department of Electrical Engineering, University of Transport \& Communications, Hanoi 100000, Vietnam

aemail: 15119005@bjtu.edu.cn, ${ }^{\mathrm{b} e m a i l: ~ h s z h a n g @ b j t u . e d u . c n, ~}{ }^{\mathrm{C} e m a i l}:$ nguyennghia.ktd@utc.edu.vn

Keywords: Phasor measurement unit (PMU); Optimal placement; Integer linear programming (ILP); Distribution system observability; Multi-configuration network

\begin{abstract}
Phasor Measurement Unit (PMU) is more and more concerned in power distribution network due to its great benefit. In near future, it may become an essential device in the modern distribution systems. The Optimal PMU Placement (OPP) problem on transmission network for full system observability, was fully done. However, in distribution network the change of configuration in normal operation and contingency modes has a very large impact to find the location of PMUs on network. Some methods have been proposed to solve this problem however the researchers had to spend lot of effort to calculate the system data in all configurations of network. This paper proposes a new solution to find the optimal location of PMUs, which can ensure the full system observability in all operation configurations of distribution network with calculating only based on one configuration. To justify the methodology of solution, the simulations on IEEE RBTS-2 bus and 33 buses distribution systems are presented for experiment. The results of this study show that the proposed solution is effective and feasible.
\end{abstract}

\section{Introduction}

Synchrophasor Measurements has become of interest in supervision of stability for power distribution network systems because of more dynamic requirements for the system caused by renewable power infeed such as wind and solar as well as energy trade [1-3]. The major limit to PMU deployment in distribution systems is the cost, but several projects have already been in progress worldwide [4]. In near future, PMU may become an essential device in the modern distribution systems. The OPP problem on transmission network for full system observability, was fully done by many different methods as Integer Linear Programming (ILP), Heuristic, or Metaheuristic [5-9]. However, in distribution network the change of configuration in operation modes has a very large impact to find the location of PMUs on network.

Abdelsalam et al. [10] investigated the impact of network reconfiguration on PMUs location, then proposed one PMU placement solution to cover fully system observability in all configurations by merging the PMU location in all configurations into one. This solution is not effective because almost all buses need to install PMU, thus leading to high costs.

A new algorithm of PMU placement for distribution networks was proposed by Emad Jamil [11]. Although the computational time of this approach may be improved, the obtained result is not as good as ILP method. Moreover, this method can use for one configuration, with multi-configuration networks it is difficult to apply.

The authors in [12] proposed an overall optimization solution to specify location of PMUs that is, each PMU is tested for all configurations and it is considered acceptable only if it allows the accuracy limits to be met in all of them. This solution can reduce the number of PMUs, it nevertheless has to scan through all configurations in which case that demands lot of effort in calculating. 
The work was extended in [13] with considering the performance degradation of measurement devices. With the aim of restricting the searching space, Liu et al. [14] developed a model for robust measurement placement with the saturate number of system. Recently, the authors in reference [15] proposed a new model for robust meter placement problem that specify the minimization of the worst case total estimation variance. However, as similar to the solution in [12] these methods still require very much effort to calculate for all network configurations.

This paper proposes a new solution to find the location of PMU, which bases on only one configuration of network. From the known location of tie switches, by closing all these switches and then applying the proposed constraint formula for bus observation in that configuration we will find out the placement of PMU, which can ensure the full system observability in all operation configurations of network. The proposed solution is tested on IEEE RBTS-2 bus and 33 bus distribution systems, and results are compared with other solutions.

\section{Theoretical Approach}

\section{Configuration change in distribution network}

The configuration of distribution network is created by the status of sectionalizing switches and tie switches. The location of these switches and the status of them in different operation conditions are specified by optimal algorithms [16-19].

The change of configuration in distribution network is the process of opening or closing status of sectionalizing switches and tie switches to transfer loads among the feeders. These switching are performed in such a way that the radiality of the network is maintained and all the loads are energized. This work can reduce the power losses, hold the balance of load, improve the power quality and increase the reliability as well as the security of system. Moreover, it also help to improve the bus voltage profile and reduce line losses, as well as avoid the overloading network branches [20].

In modern distribution network, all sectionalizing switches and tie switches are automatic switches and the configuration of power network may be controlled automatically at management centre.

\section{Power distribution network observation}

In general, the problem of network observation is divided into two categories, the numerical category and the topological category [21]. The numerical methods base on the mathematical model of power network to solve the measurement device placement problem, whereas the topological methods use the decoupled measurement model and graph theory.

Numerical methods require lot of network information such as line parameter, load and generator profile, or status of switches in each network configuration; then topological method is simpler. The objective function for full network observation is defined as follows:

$$
\min \sum_{k=1}^{n} w_{k} x_{k}
$$

Subject to $f_{i}\left(x_{k}\right)=A_{\text {config } i \cdot x_{k}} \geq 1$

where $x_{k}$ is a variable which is 0 or 1 whether PMU placed at bus or not; $w_{k}$ is the cost of the PMU installed at that bus; $n$ is number of bus; $f_{i}(x)$ is a vector function, whose entries are non-zero if the corresponding bus voltage is solvable using the given measurement; $A_{\text {config_i }}(i=1, . ., q)$ is adjacency matrix of power system in each configuration, $q$ is number of configuration. The elements of matrix $A_{\text {config_i } i}$ are defined as,

$A_{k, l}=1$ if $k=l$

$=1$ if bus $k$ is connected to bus $l$.

$=0$ otherwise.

\section{Proposed Solution}

In distribution network, there will be up to $3^{\mathrm{m}}$ configurations if the number of tie switches is $m$ 
[22]. When $m$ is a large number that is impossible to find out the placement of PMUs in each configuration and then merging all of them into one, then it needs to find another solution. Example as in Fig. 1, it supposes that the system consists of three configurations as listed as in Table 1. According to Eq. 1, we have the constraint of bus 2 as follow:

$$
\begin{aligned}
& f_{1}\left(x_{2}\right)=x_{1}+x_{2}+x_{3} \geq 1 \\
& f_{2}\left(x_{2}\right)=x_{2}+x_{3} \geq 1 \\
& f_{3}\left(x_{2}\right)=x_{1}+x_{2} \geq 1
\end{aligned}
$$

Because $x_{k}$ are binary variables so Eq. (2) can be written as:

$$
f\left(x_{2}\right)=f_{1}\left(x_{2}\right) \cdot f_{2}\left(x_{2}\right) \cdot f_{3}\left(x_{2}\right) \geq 1
$$

Substituting $f_{1}\left(x_{2}\right), f_{2}\left(x_{2}\right), f_{3}\left(x_{2}\right)$, Inequality (3) is rewritten as follows:

$$
f\left(x_{2}\right)=\left(x_{1}+x_{2}+x_{3}\right)\left(x_{2}+x_{3}\right)\left(x_{1}+x_{2}\right) \geq 1
$$

After abbreviating the inequality (4) we obtain the final irreducible constraint:

$$
f\left(x_{2}\right)=x_{2}+x_{1} x_{3} \geq 1
$$

From constraint (5), it can be seen that only one PMU installed at bus 2 or two PMUs are placed simultaneously at bus 1 and bus 3, is enough to ensure the observability for bus 2 . Although $x_{k}$ are binary variables, they are also counted as integer variables with their value from 0 to 1 . Based on this clue, because value of $x_{i}(i=0,1, \ldots, 4)$ is only 0 or 1 so $x_{1} x_{3}=1$ is equivalent to $x_{1}+x_{3}=2$, then we may rewrite (5) in algebraic form as (6).

$$
f\left(x_{2}\right)=2 x_{2}+x_{1}+x_{3} \geq 2
$$

Comparison between (5) and (6), it is clearly that the meaning is still the same.

Similarly, we can get the constraints of other buses:

$$
\begin{aligned}
& f\left(x_{0}\right)=2 x_{0}+x_{1}+x_{4} \geq 2 \\
& f(x 1)=x 0+2 x_{1}+x_{2} \geq 2 \\
& f(x 3)=x 2+2 x 3+x 4 \geq 2 \\
& f(x 4)=x 0+x 3+2 x^{2} \geq 2
\end{aligned}
$$

Table 1. 5-Bus network configurations

\begin{tabular}{c|c|c|c}
\hline & Sw1 & Tie1 & Comment \\
\hline Config 1 & close & open & Normal mode \\
\hline Config 2 & open & close & Load balancing \\
\hline Config 3 & close & close & Line 3 outage \\
\hline
\end{tabular}

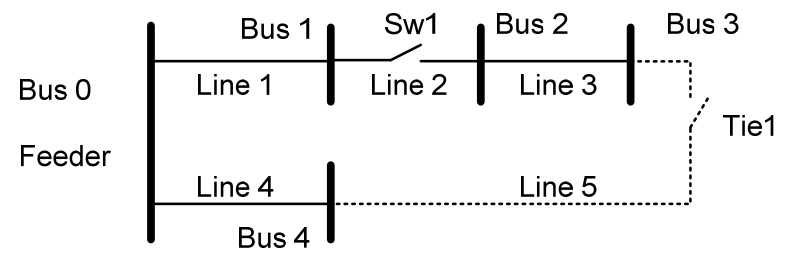

Fig. 1. A 5-bus distribution network

Distribution network is commonly radial configuration, one bus often connects to two other buses therefore inequality (6) seems to be a general form. However, when bus having many connections as illustrated as in Fig. 2, this conversion also can be applied generally.

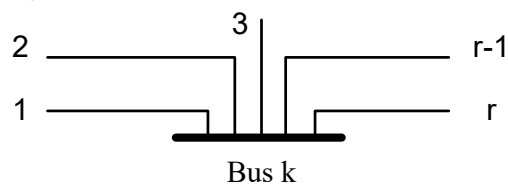

Fig. 2. Multi-connection bus

To ensure the observability for bus in any cases, the constraint will be:

$$
f\left(x_{k}\right)=x_{k}+x_{1} x_{2} x_{3} \ldots x_{r-1} x_{r} \geq 1
$$

then the conversion as follow:

$$
f\left(x_{k}\right)=r x_{k}+x_{1}+x_{2}+x_{3}+\ldots+x_{r-1}+x_{r} \geq r
$$

where, $r$ is total number of bus which has direct connection to bus $\mathrm{k}$.

In constraint inequality (9) we do not need to concern about status of sectionalizing switches or 
tie switches even the outage of lines. That means the bus observation can be remained in any configurations of distribution network.

Through above analysis, we propose a solution by applying constraint inequality (9) for network configuration when closing all sectionalizing switches and tie switches, in that case the PMU placement will ensure the full system observability in all operation configurations of power network.

Applying the above proposed solution for network in Fig. 1, closing Sw1 and Tie1, then the constraint of buses can be written as follow:

$$
\begin{aligned}
& f(x 0)=2 x 0+x 1+x 4 \geq 2 \\
& f(x 1)=x 0+2 x 1+x 2 \geq 2 \\
& f(x 2)=x 1+2 x 2+x 3 \geq 2 \\
& f(x 3)=x 2+2 x 3+x 4 \geq 2 \\
& f(x 4)=x 0+x 3+2 x 4 \geq 2
\end{aligned}
$$

These constraints are the same with constraints (6) and (7). That means the PMU placement solution for one configuration, in which case all the sectionalizing and tie switches are at closing status that maybe ensure the full system observability in all operation configurations of network.

From above analysis, the object function and constraints of OPP problem become:

$$
\begin{aligned}
& \min \sum_{k=1}^{n} w_{k} x_{k} \\
& \text { Subject to } f(x)=A . x \geq b
\end{aligned}
$$

$A$ is the connectivity matrix of power distribution system, the elements of matrix A are specified as follows:

$$
A_{k l}=\left\{\begin{array}{c}
r \text { if } k=l . \\
1 \text { if bus } k \text { is connected to bus } j . \\
0 \text { otherwise. }
\end{array}\right.
$$

Vector $\mathrm{b}$ expresses the expectation with regard to the observability for each bus.

$$
b=r
$$

Now we can use one of any methods as ILP, Heuristic or Metaheuristic to solve the OPP problem. The obtained result will satisfy the requirement of robust observability in the change of network configuration as well as in contingency modes.

\section{Simulation Results}

To experiment the proposed solution, simulations on the IEEE RBTS-2 bus and 33 bus distribution systems are carried out in MATLAB.

Fig. 3 illustrates the one line diagram of IEEE RBTS-2 bus distribution system. This network consists of 22 load buses, 5 sectionalizing switches and 2 tie switches; and the placement of PMU after applying the proposed solution is presented in Table 2. We need 11 PMUs to cover all system in any configurations. With the exception of PMU buses, all other buses are observed by two different PMUs, therefore the full system observability is still remained when switches change their status or even one line outage. 


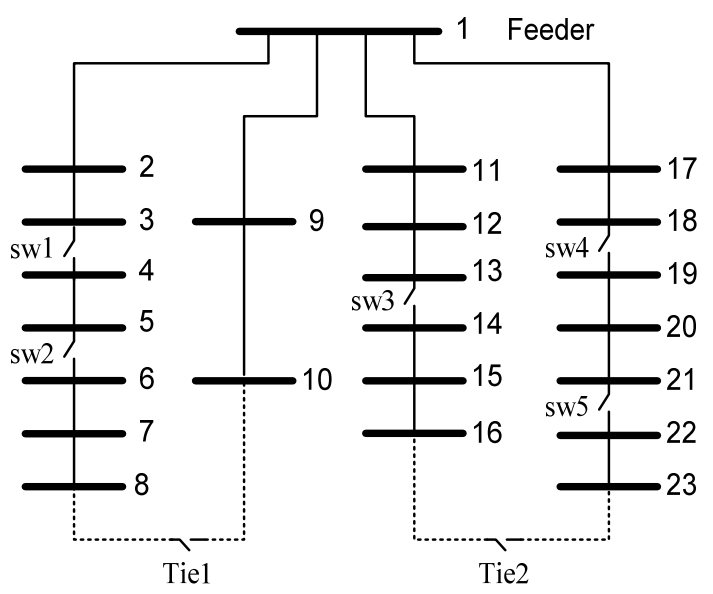

Fig. 3. Diagram of IEEE RBTS-2 bus distribution system

Table 2. Placement of PMU on RBTS-2 Bus System

\begin{tabular}{c|c}
\hline Number of Tie switch & 2 \\
\hline Number of Configuration & 9 \\
\hline Number of PMU & 11 \\
\hline PMU location & $1,3,5,7,10,12,14,16,18,20,22$ \\
\hline
\end{tabular}

In order to depict the performance of proposed solution, the result will be compared with the solution in reference [10]. There are many configurations we only give three typical configurations for experiment. The detail information of these configurations as in Table 3 then Table 4 shows the obtained result. As we can see, it needs up to 19 PMUs for covering all system in only three configurations. Moreover, it spends one time to find out the placement of PMU for one configuration, and will be $\mathrm{n}$ times for $\mathrm{n}$ configurations. That is clearly not feasible and effective when comparing to proposed solution with only one time to find out the final goal.

To illustrate more clearly the effect of proposed solution, the simulation is carried out on the 33 bus distribution system. This network contains 31 load buses, and that maybe change among 243 configurations by 11 sectionalizing switches and 5 tie switches. Fig. 4 shows the diagram of this system. The simulated result is demonstrated in Table 5. To ensure fully system observability, 17 PMUs need to be installed on network. With this PMU placement, one bus except PMU buses is all covered by at least two different PMUs, that can ensure reliably the full system observability in any configurations.

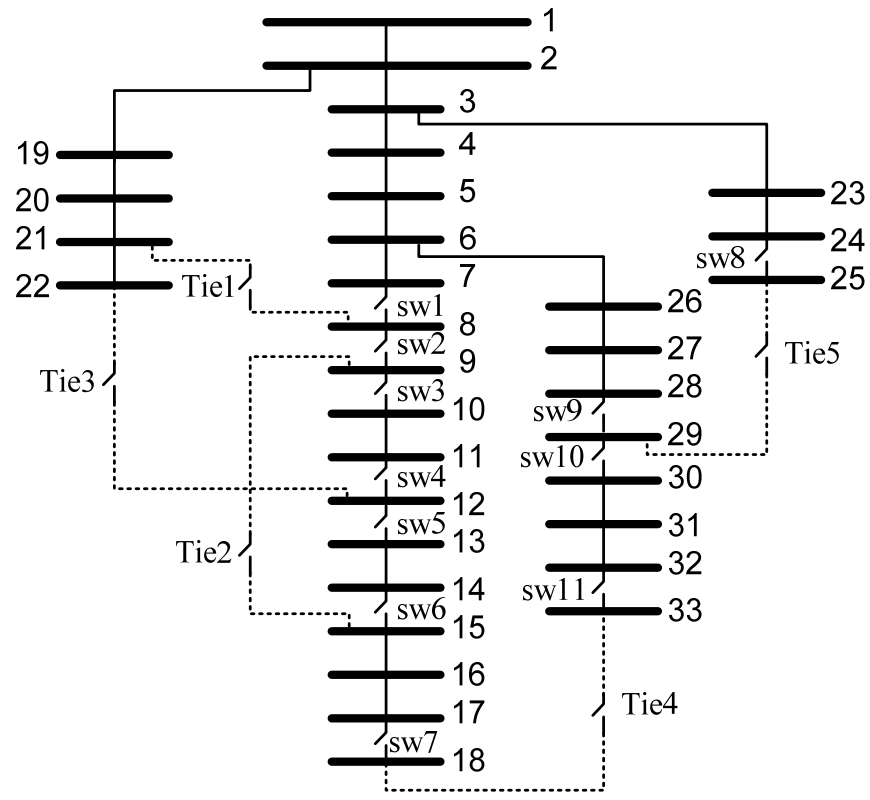

Fig. 4. Diagram of 33 buses distribution network 
Table 3. Three configurations of RBTS-2 Bus system

\begin{tabular}{c|c|c|c}
\hline & Config. 1 & Config. 2 & Config. 3 \\
\hline Sw1 & close & close & close \\
\hline Sw2 & close & open & close \\
\hline Sw3 & close & close & close \\
\hline Sw4 & close & close & close \\
\hline Sw5 & close & open & close \\
\hline Tie1 & open & close & close \\
\hline Tie2 & open & close & Load balancing \\
\hline Comment & Normal & Lines 6-7 and 15-16 outage \\
\hline
\end{tabular}

Table 4. Placement of PMU for different configurations of RBTS-2 bus

\begin{tabular}{c|c|c}
\hline & Number of PMU & PMU location \\
\hline Config. 1 & 8 & $1,4,7,10,13,16,19,22$ \\
\hline Config. 2 & 9 & $2,5,6,10,12,15,18,21,22$ \\
\hline Config. 3 & 9 & $3,4,7,9,12,15,18,20,23$ \\
\hline Merging & 19 & $1,2,3,4,5,6,7,9,11,12,13,15,16,18,19,20,21,22,23$ \\
\hline
\end{tabular}

Table 5. Placement of PMU on 33 bus distribution system

\begin{tabular}{c|c}
\hline Number of tie switch & 5 \\
\hline Number of configuration & 243 \\
\hline Number of PMU & 17 \\
\hline PMU location & $1,3,4,6,8,10,12,13,15,17,19,21,24,27,29,31,33$ \\
\hline
\end{tabular}

The simulated results on the test systems illustrate the salient effect of proposed solution. It may only know about location of tie switches on network that can find out the final goal without the load and generator profile or any specific configurations. This will save significantly the effort and time to calculate the system data in each configuration as previous methods. Based on the finding of this study, the problem of PMU placement for robust measurement in multi-configuration power distribution networks that can be solved simply and effectively.

\section{Conclusions}

This paper proposes a new solution to solve the PMU placement problem in multi-configuration distribution network. Based on the transforming of constraints of all configurations into one general form, the proposed solution can find out the final goal with calculating for only one configuration. The effect of proposed solution is demonstrated by simulations on IEEE RBTS-2 bus and 33 buses distribution systems. The results of this study show that the proposed solution is feasible and effective.

\section{Acknowledgment}

The authors of this paper would like to acknowledge the support of the Science and Technology Development Plan of China Railway (Grant 2015Z005-D) and the National Natural Science Foundation of China (Grant 61174179).

\section{References}

[1] Wache, M. and D.C. Murray. Application of Synchrophasor Measurements for distribution networks [C]. 2011 IEEE Power and Energy Society General Meeting, 2011.

[2] Ochoa, L.F. and D.H. Wilson. Using synchrophasor measurements in smart distribution networks [C]. 21st International Conference on Electricity Distribution CIRED, 2011.

[3] Muscas, C., et al., Smart electric energy measurements in power distribution grids [J]. IEEE Instrumentation \& Measurement Magazine, 2015 18(1) 17-21. 
[4] Meier, A.v., et al. Micro-Synchrophasors for Distribution Systems [C]. IEEE Power \& Energy Society Innovative Smart Grid Technologies Conference, 2014.

[5] $\mathrm{Xu}, \mathrm{B}$. and A. Abur, Observability Analysis and Measurement Placement for Systems with PMUs [C]. Power Systems Conference \& Exposition, 2004. 943-946.

[6] Gou, B., Optimal Placement of PMUs by Integer Linear Programming [J]. IEEE Transaction on Power Systems, 2008 23( 3) 1525-1526.

[7] Shewale, R.H., et al., Optimal placement of phasor measurement unit for power system observability by heuristic search method [J]. International journal of Advanced Technology \& Engineering Research, 2012 2(2) 128-133.

[8] Aghaei, J., et al., Multi-objective Phasor Measurement Unit Placement in Electric Power Networks: Integer Linear Programming Formulation [J]. Electric Power Components \& Systems, 2015 43(17) 1902-1911.

[9] Babu, R. and B. Bhattacharyya, Optimal allocation of phasor measurement unit for full observability of the connected power network [J]. Electrical Power and Energy Systems, 201679 89-97.

[10] Abdelsalam, H.A., A.Y. Abdelaziz, and V. Mukherjee. Optimal PMU placement in a distribution network considering network reconfiguration [C]. International Conference on Circuit, Power and Computing Technologies, 2014.

[11] Jamil, E., M. Rihan, and M.A. Anees. Towards optimal placement of phasor measurement units for smart distribution systems [C]. Power India International Conference (PIICON), 2014 6th IEEE, 2014. Delhi.

[12] Liu, J., et al., Trade-Offs in PMU Deployment for State Estimation in Active Distribution Grids [J]. IEEE Transactions on Smart Grid, 2012 3(2) 915-924.

[13] Liu, J., et al. Optimal placement for robust distributed measurement systems in active distribution grids [C]. IEEE International Instrumentation \& Measurement Technology Conference, 2013.

[14] Wang, H., et al. A robust measurement placement method in distribution system state estimation [C]. 2014 IEEE PES Asia-Pacific Power and Energy Engineering Conference (APPEEC), 2014.

[15] Damavandi, M.G. and V. Krishnamurthy, Robust Meter Placement for State Estimation in Active Distribution Systems [J]. IEEE Transactions on Smart Grid, 2015 6(4) 1972-1982.

[16] Celli, G. and F. Pilo, Optimal sectionalizing switches allocation in distribution networks [J]. IEEE Transactions on Power Delivery, 1999 14(3) 1167-1172.

[17] Lim, I.-H., et al., An Algorithm on Optimal Placement Decision of Automatic Switches for 6 Sections/3 Links Configuration in DAS [J]. Journal of Electrical Engineering \& Technology, 2011 6(3) 328-333.

[18] Ray, S., A. Bhattacharya, and S. Bhattacharjee, Optimal placement of switches in a radial distribution network for reliability improvement [J]. Electrical Power and Energy Systems, 201676 53-68.

[19] Baran, M.E. and F.F. Wu, Network reconfiguration in distribution systems for loss reduction and load balancing [J]. IEEE Transactions on Power Delivery, 1999 4(2) 1401-1407.

[20] Mishra, S., D. Das, and S. Paul, A comprehensive review on power distribution network reconfiguration [J]. Energy Systems, 2016 1-58.

[21] MohammadiIvatloo, B., Optimal Placement of PMUs for Power System Observability Using Topology Based Formulated Algorithms [J]. Journal of Applied Sciences, 2009 9(13) 2463-2468.

[22] Abul'Wafa, A.R., A new heuristic approach for optimal reconfiguration in distribution systems [J]. Electric Power Systems Research, 201181 282-289. 